Original Research Paper

\title{
Variants Within over a Hundred Complete COVID-19 Genomes and the Impact on Health Security
}

\author{
Sayed A.M. Amer \\ Department of Forensic Sciences, College of Criminal Justice, Naif Arab University for Security Sciences, Saudi Arabia
}

\section{Article history}

Received: 04-10-2020

Revised: 21-01-2021

Accepted: 22-01-2021

Email: samer@nauss.edu.sa

\begin{abstract}
COVID-19 genomes have been collected from the viral genomes database to track and characterize novel variants. The data were treated bioinformatically so that 172 variants, with 127 unique and 45 polymorphic variants were found. The 127 unique variants consist of 76 missense, 39 synonymous, 6 non-coding, 5 deletions and 1 insertion. The 45 polymorphic variants consist of 25 missense, 15 synonymous, 4 non-coding and 1 in-frame-deletion. Most common variants are $28144 \mathrm{~T}>\mathrm{C} \quad(33$ missense), $8782 \mathrm{C}>\mathrm{T} \quad(31$ synonymous), followed by missense $11083 \mathrm{G}>\mathrm{T}$ (11 samples), $18060 \mathrm{C}>\mathrm{T}$ (9 samples) and 26144G $>\mathrm{T}$ (7 samples). L3606F, S5932F and L84S are the amino acid changes in the last three common variants. Most variants were found in ORF1ab gene within the region encoded for domains (nsp4 and nsp6) and in the coding ORF8 gene. The variant $28144 \mathrm{~T}>\mathrm{C}$ could be among the main enhancers of viral transmission. There is a tendency for a national specificity of the most recorded variants. The virus outbreak could be between countries or dependent on the place of origin. Reasonable evidence of Chinese origin of the virus could be possible and thus more genomes should be collected and analyzed to understand the origin and the reason for its outbreak. This could support human health security by either finding out suitable vaccines or managing health precautionary measures.
\end{abstract}

Keywords: Coronavirus, Genome, Variants, Mutation, Health Security

\section{Introduction}

Since the virus is like SARS-CoVs, the International Committee on Taxonomy of Viruses (ICTV) termed the newly discovered Coronavirus (2019-nCoV) SARS-CoV-2 (Cascella et al., 2020). It was first reported in Wuhan, the largest metropolitan area in China on December 31st, 2019. Therefore, the (WHO, 2020) acronymized this 2019 disease as "COVID-19". CoVs can infect different species including birds, mice, bats, livestock and humans (Wang et al., 2006; Ge et al., 2013; Chen and Guo, 2016), with a serious infection in humans, causing pneumonia.

Systematically, CoVs belong to the subfamily Orthocoronavirinae of the family Coronaviridae and the order Nidovirales. Coronavirinae contains four genera: Alphacoronavirus, Betacoronavirus (4 subgenera) (Chan et al., 2013), Gammacoronavirus and Deltacoronavirus (Chen et al., 2020). Bats and rodents are the probable source of alpha and Betacoronaviruses, whereas birds could be the source of Gamma and delta viruses (Cascella et al., 2020). Over 210,000 COVID-19 complete genomes have so far been sequenced with an approximate genome length of 30000 bases. As fast as the virus spreads, more variants accumulate with the possibility of further emerging virulent strains. The virus genome is found in a single-stranded positive-sense RNA (+ssRNA) (Perlman and Netland, 2009) acquiring 5'-cap structure and 3'-poly-A tail (Chen et al., 2020). As a severe global health threat, CoVs outbreaks are, most probably, unavoidable in the future. There is an urgent need, thus, to identify the possible variants in the 102 sequenced genomes that might support in understanding the reasons for the virus outbreak and in producing an effective therapy and vaccine against the virus.

\section{Materials and Methods}

The COVID-19 page (https://bigd.big.ac.cn/ncov) available in China's National Genomics Data Center (NGDC) was accessed on the 27th of September 2020. 
From 2019-nCoV genomes available on this page and in three Genbank databases, 102 publicly complete genomes have been collected and are listed in Table 1. As has been previously used (Koyama et al., 2020; Matsuda et al., 2020), the two longest and identical NC_045512 and MN908947 sequences (29903 bp) were used as reference genomes to coordinate the differences in the ribosomal slippage of the 102 used genomes. The genome data were checked for the accuracy of the alignments and the aligned data could be obtained from the author upon request. Since genomes acquire differences in their start and end points, their lengths have been adjusted to the lengths of NC_045512 and MN908947 and their variants were numbered according to the positions of these two genomes. All genomes were first aligned to each other using BioEdit Sequence Alignment Editor (Hall et al., 2011) and the FASTA file of the aligned data was executed to MacClade v.4.10 program (Maddison and Maddison, 2002) and manually aligned to the reference genomes. Using MacClade v.4.10, mutations were recorded and data were transferred to the Paup v. 4.0b10 (Swofford, 2002) program for phylogenetic analysis.

After deleting ambiguous, gap-containing sites and $\mathrm{N}$ or mixed bases sites, the remaining 29532 sites were analyzed by Maximum-Parsimony (MP), NeighborJoining (NJ) and Maximum-Likelihood (ML) methods in Paup v. 4.0b10. For MP, heuristic searches of 10 random stepwise additions were conducted by Tree Bisection Reconnection (TBR) branch swapping and 1000 bootstrap replications. For NJ analysis (Saitou and Nei, 1987; Tamura and Nei, 1993) distance option and 1000 bootstrap replications were used. As the MT226610 sequence was rapidly evolved, it was used as an outgroup. For ML, heuristic searches by axis additions and NearestNeighbor Interchange (NNI) branch-swapping were adjusted. Other conditions for the ML analysis like gamma shape parameter of 1.5214 and 4 rate categories were also adjusted. The substitution rate matrix of the data was as follow: $\mathrm{R}(\mathrm{a})=0.1571, \mathrm{R}(\mathrm{b})=0.7500, \mathrm{R}(\mathrm{c})=$ $0.2121, \mathrm{R}(\mathrm{d})=0.6049, \mathrm{R}(\mathrm{e})=2.6308$ and $\mathrm{R}(\mathrm{f})=1.00$. Likelihood settings from best-fit model (GTR+I+G) were selected by AIC in Modeltest Version 3.06.

Table 1: Organization of the reference + ssRNA genome used in this study. Nsp $=$ non structural protein; ORF $=$ Open Reading

\begin{tabular}{|c|c|c|c|}
\hline Gene & Position & Product & Notes \\
\hline 5'UTR & $1-265$ & 5'UTR & cap \\
\hline \multirow[t]{18}{*}{ Orf1ab } & $266-805$ & nsp1 & Leader protein \\
\hline & $806-2719$ & nsp2 & \\
\hline & $2720-8554$ & nsp3 & \\
\hline & $8555-10054$ & nsp4 & Transmembrane domain 2 \\
\hline & $10055-10972$ & nsp5 & 3C-like proteinase \\
\hline & $10973-11842$ & nsp6 & Putative transmembrane domain \\
\hline & $11843-12901$ & nsp7 & \\
\hline & $12092-12685$ & nsp8 & \\
\hline & 12686-13042 & nsp9 & ssRNA-binding protein \\
\hline & $13025-13441$ & nsp10 & Growth-factor-like protein \\
\hline & $13442-13480$ & nsp11 & \\
\hline & $13476-13503$ & Stem_loop & Frameshifting stimulation element \\
\hline & $13488-13542$ & Stem_loop & Frameshifting stimulation element \\
\hline & $13442-13468,13468-16235$ & nsp12 & RNA-dependent RNA polymerase \\
\hline & $16237-18093$ & nsp13 & Helicase \\
\hline & $18040-19620$ & nsp14 & 3'-to-5' exonuclease \\
\hline & $19621-20658$ & nsp15 & endoRNAse \\
\hline & 20659-21552 & nsp16 & O-ribose methyltransferase \\
\hline $\mathrm{S}$ & $21563-25384$ & spike glycoprotein & Surface glycoprotein \\
\hline ORF3a & $25393-26220$ & ORF3a protein & \\
\hline $\mathrm{E}$ & $26245-26472$ & envelope protein & \\
\hline M & $26523-27191$ & membrane glycoprotein & \\
\hline ORF6 & $27202-27387$ & ORF6 protein & \\
\hline ORF7a & $27394-27759$ & ORF7a protein & \\
\hline ORF7b & $27756-27887$ & ORF7b & \\
\hline ORF8 & 27894-28259 & ORF8 protein & \\
\hline $\mathrm{N}$ & 28274-29533 & nucleocapsid phosphoprotein & ORF9 protein \\
\hline ORF10 & $29558-29674$ & ORF10 protein & \\
\hline ORF10 & 29609-29644 & -- & 3'UTR pseudoknot stem-loop 1 \\
\hline ORF10 & $29629-29657$ & -- & 3' UTR pseudoknot stem-loop 2 \\
\hline 3'UTR & $29675-29903$ & 3'UTR & tail \\
\hline
\end{tabular}




\section{Results and Discussion}

The COVID-19 genome is approximately $30,000 \mathrm{bp}$. It contains within its 5'-proximal two-thirds, nonstructural protein coding regions (nsp1-nsp16; replicase genes) encoded on orf1ab gene. Within the 3'-proximal one-third of the genome, structural proteins and nonessential accessory protein coding regions are encoded (ORF3ORF10, S, E, M, N) (Paul, 2006). The 5' UTR (265 b) comprises the genome cap and the 3' UTR ( 300 b) forms the tail (Table 1). The recorded variants were counted between positions 33 and 29830 to avoid base bias. The mixed bases were not considered in the analysis. 172 total variants are found, 127 unique and 45 polymorphic (Table 2). The unique variants (Table 3 ) are 121 substitutions (76 missense, 39 synonymous and 6 non-coding), 5 deletions ( 2 in-frame-deletions, 2 out-of-frame-deletions and 1 noncoding) and 1 insertion. The polymorphic variants (Table 4) are 45 substitutions ( 25 missense, 15 synonymous, 4 non-coding and 1 in-frame-deletion) (Fig. 1).
In agreement with previous studies (Koyama et al., 2020; Matsuda et al., 2020), most common variants were $8782 \mathrm{C}>\mathrm{T}$ (ORF1ab) and 28144T $>\mathrm{C}$ (ORF8) in 31 samples followed by $11083 \mathrm{G}>\mathrm{T}$ (nsp6) in 11 samples and $18060 \mathrm{C}>\mathrm{T}$ (nsp14) in 9 samples. The occurrences of $8782 \mathrm{C}>\mathrm{T}$ and $28144 \mathrm{~T}>\mathrm{C}$ concur and most of the other common variants are subsets of these most common ones. $8782 \mathrm{C}>\mathrm{T}$ is synonymous; however, $11083 \mathrm{G}>\mathrm{T}$ (L3606F) and $18060 \mathrm{C}>\mathrm{T} \quad(\mathrm{S} 5932 \mathrm{~F})$ and $28144 \mathrm{~T}>\mathrm{C}(\mathrm{L} 84 \mathrm{~S})$ are missense causing amino acid changes in nsp6, nsp14 and ORF8, respectively. The variant $28144 \mathrm{~T}>\mathrm{C}$ which exhibited an amino acid change in ORF8 protein from Leucine to Sereine, was indicated in the polypeptide involved in enhancing virus transition from bat to human (Chan et al., 2020; Nguyen et al., 2020). As this variant was recorded in thirteen North Americans, ten central Asians (Chinese including Wuhan residents), three Japanese, one Taiwanese, one Indian and one Spanish, it could be among the main enhancers of viral transmission.

Table 2: Statistics of the 172 recorded mutations in the 102 COVID-19 genomes

\begin{tabular}{|c|c|c|c|c|c|c|c|}
\hline \multirow[b]{2}{*}{ Variants } & \multirow{2}{*}{$\begin{array}{l}\text { Substitution } \\
- \text { Missense } \\
\end{array}$} & \multicolumn{2}{|l|}{ Deletion } & \multirow[b]{2}{*}{ In-frame-deletion } & \multirow[b]{2}{*}{ Out-of-frame deletion } & \multirow[b]{2}{*}{ Non-coding } & \multirow[b]{2}{*}{ Insertion } \\
\hline & & Synonymous & Non-coding & & & & \\
\hline Unique & 76 & 39 & 6 & 2 & 2 & 1 & 1 \\
\hline Polymorphic & 25 & 15 & 4 & 1 & - & - & - \\
\hline
\end{tabular}

Table 3: Unique monomorphic variants (substitution, deletion and insertion), their positions, number of cases and country distribution found in the $102 \mathrm{CoVs}$ genomes

\begin{tabular}{|c|c|c|c|c|}
\hline$\#$ & Variant & Type & Amino acid change & Accession number, country \\
\hline 1 & $35 \mathrm{~A}>\mathrm{T}$ & Non-coding & & MT163716, USA \\
\hline 2 & $36 \mathrm{C}>\mathrm{T}$ & Non-coding & & MT163716, USA \\
\hline 3 & $75 \mathrm{C}>\mathrm{A}$ & Non-coding & & MT049951, CHN \\
\hline 4 & $186 \mathrm{C}>\mathrm{T}$ & Non-coding & & MT093631, CHN \\
\hline 5 & $382 \mathrm{~A}>\mathrm{T}$ & Synonymous & & LC521925, JP \\
\hline 6 & $359-382$ & In-frame-deletion & G32_V39 & LC521925, JP \\
\hline 7 & $490 \mathrm{~T}>\mathrm{A}$ & Synonymous & & MT044257, USA \\
\hline 8 & $514 \mathrm{~T}>\mathrm{C}$ & Synonymous & & MT188340, USA \\
\hline 9 & $654 \mathrm{G}>\mathrm{A}$ & Synonymous & & MT123293, CHN \\
\hline 10 & $686-694$ & In-frame-deletion & K141_F143 & MT044258, USA \\
\hline 11 & $1102 \mathrm{C}>\mathrm{T}$ & Synonymous & & MT188339, CHN \\
\hline 12 & $1348 \mathrm{C}>\mathrm{T}$ & Synonymous & & GWHACDD01000001, PAK \\
\hline 13 & $1385 \mathrm{C}>\mathrm{T}$ & Missense & $\mathrm{H} 374 \mathrm{Y}$ & MT159720, USA \\
\hline 14 & $1397 \mathrm{G}>\mathrm{A}$ & Synonymous & & GWHACDD01000001, PAK \\
\hline 15 & $1548 \mathrm{G}>\mathrm{A}$ & Missense & S428N & MN994467, USA \\
\hline 16 & $1912 \mathrm{C}>\mathrm{T}$ & Synonymous & & LC521925, JP \\
\hline 17 & $2091 \mathrm{C}>\mathrm{T}$ & Missense & T609I & MT027064, USA \\
\hline 18 & $2269 \mathrm{~A}>\mathrm{T}$ & Synonymous & & MT066156, ITA \\
\hline 19 & $2277 \mathrm{~T}>\mathrm{C}$ & Missense & I671T & MT012098, IND \\
\hline 20 & $2446 \mathrm{~T}>\mathrm{C}$ & Synonymous & & MT163716, USA \\
\hline 21 & $2717 \mathrm{G}>\mathrm{A}$ & Missense & G818S & MT093571, SWE \\
\hline 22 & $2971 G>T$ & Missense & M902I & MT049951, CHN \\
\hline 23 & $3037 \mathrm{C}>\mathrm{T}$ & Synonymous & & MT192765, USA \\
\hline 24 & $3177 \mathrm{C}>\mathrm{T}$ & Missense & P971L & MT044257, USA \\
\hline 25 & $3259 \mathrm{G}>\mathrm{T}$ & Missense & T998G & MT159707, USA \\
\hline 26 & $3411 \mathrm{C}>\mathrm{T}$ & Missense & E1049V & MT163716, USA \\
\hline 27 & $3738 \mathrm{C}>\mathrm{T}$ & Missense & P1158L & MT159705, USA \\
\hline 28 & $3792 \mathrm{C}>\mathrm{T}$ & Missense & E1176V & LC522973, JP \\
\hline
\end{tabular}




\section{Table 3: Continue}

\begin{tabular}{|c|c|c|c|c|}
\hline 29 & $4288 \mathrm{G}>\mathrm{T}$ & Synonymous & & MT226610, CHN \\
\hline 30 & $4307 \mathrm{~A}>\mathrm{C}$ & Missense & G1348T & MT226610, CHN \\
\hline 31 & $5572 \mathrm{G}>\mathrm{T}$ & Synonymous & & MT163716, USA \\
\hline 32 & $5784 \mathrm{C}>\mathrm{T}$ & Synonymous & & MT152824, USA \\
\hline 33 & $5845 \mathrm{~A}>\mathrm{T}$ & Missense & K1860N & MT159715, USA \\
\hline 34 & $6031 \mathrm{C}>\mathrm{T}$ & Synonymous & & MT039890, SKorea \\
\hline 35 & $6035 \mathrm{~A}>\mathrm{G}$ & Missense & S1924K & MT188341, USA \\
\hline 36 & $6636 \mathrm{C}>\mathrm{T}$ & Missense & $\mathrm{T} 2124 \mathrm{I}$ & MT159712, USA \\
\hline 37 & $6695 \mathrm{C}>\mathrm{T}$ & Missense & $\mathrm{P} 2144 \mathrm{~S}$ & MT012098, IND \\
\hline 38 & $6968 \mathrm{C}>\mathrm{A}$ & Missense & L2235M & CNA0007332, Wuhan \\
\hline 39 & $6996 \mathrm{~T}>\mathrm{C}$ & Missense & $\mathrm{I} 2244 \mathrm{~T}$ & MT123293, CHN \\
\hline 40 & $7479 A>G$ & Missense & $\mathrm{N} 2405 \mathrm{~S}$ & MT226610, CHN \\
\hline 41 & $9034 \mathrm{~A}>\mathrm{G}$ & Synonymous & & MT066176, TWN \\
\hline 42 & $9157 \mathrm{~T}>\mathrm{C}$ & Missense & P2965L & MT184910, USA \\
\hline 43 & $9274 A>G$ & Synonymous & & MT093571, SWE \\
\hline 44 & $9474 C>T$ & Missense & A3070V & MT159706, USA \\
\hline 45 & $9491 \mathrm{C}>\mathrm{T}$ & Missense & $\mathrm{H} 3076 \mathrm{Y}$ & MT066176, TWN \\
\hline 46 & $9561 \mathrm{C}>\mathrm{T}$ & Missense & S3099L & MN975262, CHN \\
\hline 47 & $9924 \mathrm{C}>\mathrm{T}$ & Missense & A3220V & MT118835, USA \\
\hline 48 & $10036 \mathrm{C}>\mathrm{T}$ & Synonymous & & MT159708, USA \\
\hline 49 & $10507 \mathrm{C}>\mathrm{T}$ & Synonymous & & MT184911, USA \\
\hline 50 & $11207 \mathrm{G}>\mathrm{C}$ & Missense & F3648V & MT226610, CHN \\
\hline 51 & $11233 \mathrm{~T}>\mathrm{G}$ & Synonymous & & MT226610, CHN \\
\hline 52 & $11557 \mathrm{G}>\mathrm{T}$ & Missense & E3764D & LC522972, JP \\
\hline 53 & $11750 \mathrm{C}>\mathrm{T}$ & Missense & L3829F & MT159712, USA \\
\hline 54 & $11764 \mathrm{~T}>\mathrm{G}$ & Missense & N3833K & CNA0007332, Wuhan \\
\hline 55 & $11956 \mathrm{C}>\mathrm{T}$ & Synonymous & & MT159712, USA \\
\hline 56 & $12041 \mathrm{G}>\mathrm{C}$ & Missense & D3926H & MT226610, CHN \\
\hline 57 & $12115 \mathrm{C}>\mathrm{T}$ & Synonymous & & MT039890, SKorea \\
\hline 58 & $12160 \mathrm{G}>\mathrm{C}$ & Synonymous & & MT226610, CHN \\
\hline 59 & $12202 \mathrm{G}>\mathrm{C}$ & Synonymous & & MT226610, CHN \\
\hline 60 & $12208 \mathrm{G}>\mathrm{T}$ & Synonymous & & MT226610, CHN \\
\hline 61 & $12355 \mathrm{G}>\mathrm{C}$ & Missense & T4030K & MT226610, CHN \\
\hline 62 & $12378 \mathrm{G}>\mathrm{A}$ & Missense & $\mathrm{R} 4038 \mathrm{~K}$ & MT226610, CHN \\
\hline 63 & $12464 \mathrm{G}>\mathrm{T}$ & Missense & A4067S & MT226610, CHN \\
\hline 64 & $12467 \mathrm{G}>\mathrm{T}$ & Missense & A4068S & MT226610, CHN \\
\hline 65 & $12491 \mathrm{C}>\mathrm{T}$ & Synonymous & & MT226610, CHN \\
\hline 66 & $12514 \mathrm{G}>\mathrm{C}$ & Synonymous & & MT226610, CHN \\
\hline 67 & $12534 \mathrm{C}>\mathrm{T}$ & Missense & T4090I & MT123292, CHN \\
\hline 68 & $12572 \mathrm{G}>\mathrm{T}$ & Missense & D4103Y & MT226610, CHN \\
\hline 69 & $12578 \mathrm{G}>\mathrm{T}$ & Missense & D4015Y & MT226610, CHN \\
\hline 70 & $12582 \mathrm{G}>\mathrm{T}$ & Missense & S4106I & MT226610, CHN \\
\hline 71 & $12600 \mathrm{G}>\mathrm{A}$ & Missense & S4112N & MT226610, CHN \\
\hline 72 & $12660 \mathrm{G}>\mathrm{C}$ & Missense & $\mathrm{T} 4132 \mathrm{R}$ & MT226610, CHN \\
\hline 73 & $12685 \mathrm{G}>\mathrm{C}$ & Missense & $\mathrm{T} 4140 \mathrm{~K}$ & MT226610, CHN \\
\hline 74 & $12773 \mathrm{G}>\mathrm{T}$ & Missense & F4170S & MT226610, CHN \\
\hline 75 & $12793 \mathrm{G}>\mathrm{T}$ & Missense & $\mathrm{K} 4176 \mathrm{~N}$ & MT226610, CHN \\
\hline 76 & $13072 \mathrm{C}>\mathrm{T}$ & Synonymous & & MT123292, CHN \\
\hline 77 & $13225 \mathrm{C}>\mathrm{G}$ & Synonymous & & MT093571, SWE \\
\hline 78 & $13226 \mathrm{~T}>\mathrm{C}$ & Missense & F4321L & MT093571, SWE \\
\hline 79 & $14657 \mathrm{C}>\mathrm{T}$ & Missense & L4798F & MT012098, IND \\
\hline 80 & $15597 \mathrm{~T}>\mathrm{C}$ & Missense & M5111T & MT039890, SKorea \\
\hline 81 & $15607 \mathrm{~T}>\mathrm{C}$ & Synonymous & & MN975262, CHN \\
\hline 82 & $16467 \mathrm{~A}>\mathrm{G}$ & Missense & H5401R & MT188341, USA \\
\hline 83 & $17000 \mathrm{C}>\mathrm{T}$ & Missense & H5579Y & MN994468, USA \\
\hline 84 & $17247 \mathrm{~T}>\mathrm{C}$ & Missense & V5661A & MT126808, BRA \\
\hline 85 & $17376 A>G$ & Missense & Q5704R & MT093571, SWE \\
\hline 86 & $18512 \mathrm{C}>\mathrm{T}$ & Missense & L6083F & LC521925, JP \\
\hline 87 & $18603 \mathrm{~T}>\mathrm{T}$ & Missense & M6113T & MT106054, USA \\
\hline 88 & $18814 \mathrm{C}>\mathrm{T}$ & Synonymous & & MT192765, USA \\
\hline
\end{tabular}


Table 3: Continue

\begin{tabular}{|c|c|c|c|c|}
\hline 89 & $18975 \mathrm{~T}>\mathrm{A}$ & Missense & F6237Y & MT106054, USA \\
\hline 90 & $19065 \mathrm{~T}>\mathrm{C}$ & Missense & L6267P & MT007544, AUSTR \\
\hline 91 & $19175 \mathrm{~A}>\mathrm{C}$ & Missense & M6304L & MT106054, USA \\
\hline 92 & $19610 \mathrm{C}>\mathrm{T}$ & Missense & T6449R & MT123291, CHN \\
\hline 93 & $20281 \mathrm{~T}>\mathrm{C}$ & Synonymous & & MT163719, USA \\
\hline 94 & $20299-20301$ & Out-of-frame deletion & N6678N (nsp15) & MT039887, USA \\
\hline 95 & $20670 \mathrm{G}>\mathrm{A}$ & Missense & $\mathrm{R} 6802 \mathrm{H}$ & NMDC60013002-10, Wuhan \\
\hline 96 & $20679 \mathrm{G}>\mathrm{A}$ & Missense & R6805Q & NMDC60013002-10, Wuhan \\
\hline 97 & $20936 \mathrm{C}>\mathrm{T}$ & Missense & R6891C & MT039890, SKorea \\
\hline 98 & $20980 \mathrm{G}>\mathrm{C}$ & Missense & Q6905M & MT226610, CHN \\
\hline 99 & $21147 \mathrm{~T}>\mathrm{C}$ & Missense & L6961S & MT188339, CHN \\
\hline 100 & $21647 \mathrm{~T}>\mathrm{A}$ & Missense & Y23N & MT049951, CHN \\
\hline 101 & $21707 \mathrm{C}>\mathrm{T}$ & Missense & $\mathrm{H} 44 \mathrm{Y}$ & MT027064, USA \\
\hline 102 & $21784 \mathrm{~T}>\mathrm{A}$ & Missense & F152L & MT226610, CHN \\
\hline 103 & 21386-21388 & Insertion & S7041 & MT188341, USA \\
\hline 104 & 21997-21999 & Out-of-frame deletion & Y144Y (S gene) & MT012098, IND \\
\hline 105 & $22033 \mathrm{C}>\mathrm{A}$ & Missense & G176V & MT159716, USA \\
\hline 106 & $22104 \mathrm{G}>\mathrm{T}$ & Synonymous & & MT184910, USA \\
\hline 107 & $22224 \mathrm{C}>\mathrm{G}$ & Missense & S216F & MT039890, SKorea \\
\hline 108 & $22303 \mathrm{~T}>\mathrm{G}$ & Missense & S242R & MT007544, AUSTRALIA \\
\hline 109 & $22432 \mathrm{C}>\mathrm{T}$ & Synonymous & & MT049951, CHN \\
\hline 110 & $22785 \mathrm{G}>\mathrm{T}$ & Missense & R403M & MT012098, IND \\
\hline 111 & $23185 \mathrm{C}>\mathrm{T}$ & Synonymous & & MT188341, USA \\
\hline 112 & $23403 \mathrm{~A}>\mathrm{G}$ & Synonymous & & MT192765, USA \\
\hline 113 & $23955 \mathrm{~T}>\mathrm{G}$ & Missense & F792Y & MT093571, SWE \\
\hline 114 & $25775 \mathrm{G}>\mathrm{T}$ & Missense & C120L & MT039890, SKorea \\
\hline 115 & $26354 \mathrm{~T}>\mathrm{A}$ & Missense & L22G & MT039890, SKorea \\
\hline 116 & $27493 \mathrm{C}>\mathrm{T}$ & Missense & P61S & NMDC60013002-09, Wuhan \\
\hline 117 & $27925 \mathrm{C}>\mathrm{T}$ & Missense & $\mathrm{T} 18 \mathrm{M}$ & MT106054, USA \\
\hline 118 & $28253 \mathrm{C}>\mathrm{T}$ & Synonymous & & NMDC60013002-09, Wuhan \\
\hline 119 & $28409 \mathrm{C}>\mathrm{T}$ & Missense & $\mathrm{P} 47 \mathrm{~S}$ & MT159718, USA \\
\hline 120 & $28792 \mathrm{~A}>\mathrm{T}$ & Synonymous & & MN994467, USA \\
\hline 121 & $28878 \mathrm{G}>\mathrm{A}$ & Missense & S203N & MT106052, USA \\
\hline 122 & $28916 \mathrm{G}>\mathrm{A}$ & Missense & $\mathrm{G} 216 \mathrm{~S}$ & MT188339, USA \\
\hline 123 & $29230 \mathrm{C}>\mathrm{T}$ & Synonymous & & MT159720, USA \\
\hline 124 & $29301 \mathrm{~A}>\mathrm{T}$ & Missense & D344V & MT135043, CHN \\
\hline 125 & $29705 \mathrm{G}>\mathrm{T}$ & Non-coding & & LC522973, JP \\
\hline 126 & $29742 \mathrm{G}>\mathrm{A}$ & Non-coding & & MT106052, USA \\
\hline 127 & $29750-29759$ & Non-coding del & & MT007544 (AUSTRALIA) \\
\hline
\end{tabular}

Table 4: Polymorphic variants (substitutions), their positions, number of cases and country distribution found in the $102 \mathrm{CoVs}$ genomes

\begin{tabular}{llllll}
\hline$\#$ & variant & Type & aa change & gene & Accession number (country) \\
\hline 1 & $241 \mathrm{C}>\mathrm{T}$ & Non-coding & & $5^{\prime}$ UTR & GWHACDD01000001(PAK), MT192765(USA) \\
2 & $254 \mathrm{C}>\mathrm{T}$ & Non-coding & & $5^{\prime}$ UTR & MT184910, MT184908 (USA) \\
3 & $508-522$ & In-frame-deletion G82_V86 & orf1ab & MT044258, MT159716 (USA) \\
4 & $614 \mathrm{G}>\mathrm{A}$ & Missense & A117T & & MT027062, MT027063 (USA) \\
5 & $1691 \mathrm{~A}>\mathrm{G}$ & Missense & I476V & & MT027063 (USA), MT050493 (IND) \\
6 & $2662 \mathrm{C}>\mathrm{T}$ & Synonymous & & LC522973-LC522975 (JP) \\
7 & $3099 \mathrm{C}>\mathrm{T}$ & Missense & D954I & MT159717, MT184912 (USA) \\
8 & $4402 \mathrm{~T}>\mathrm{C}$ & Synonymous & & MT135041-MT135044 (CHN) \\
9 & $5062 \mathrm{G}>\mathrm{T}$ & Missense & L1599A & MT135041-MT135044 (CHN) \\
10 & $5084 \mathrm{~A}>\mathrm{G}$ & Missense & I1607V & MT027062, MT027063 (USA) \\
11 & $6501 \mathrm{C}>\mathrm{T}$ & Missense & P2079L & MT027063 (USA), MT050493 (IND) \\
12 & $6819 \mathrm{G}>\mathrm{T}$ & Missense & S2185I & MT123293, MT123291(CHN) \\
13 & $8782 \mathrm{C}>\mathrm{T}$ & Synonymous & & MN938384, MN975262, MT049951, MT123292, MT226610, MT135041-4, \\
& & & & GWHABKI000000004(CHN), MN985325, MN997409, MT020880, MT020881, \\
& & & & MT044257, MT106052, MT106054, MT152824, MT163717-19, MT188339, \\
& & & & MT188341 (USA), MT066175 (TAW), LC522973-75(JP), MT050493, MT050493 \\
14 & $9477 \mathrm{~T}>\mathrm{A}$ & Missense & F3071Y & (IND), MT198651, MT198652(ESP) \\
15 & $10232 \mathrm{C}>\mathrm{T}$ & Missense & R3323C & MT198651, MT198652(ESP) \\
16 & $11083 \mathrm{G}>\mathrm{T}$ & Missense & L3606F & MT192772, MT1927739 (Vietnam) \\
& & & & LC528232-33 (JP), MT126808 (BRA), MT163716, MN997409, MT184910-13 (USA), \\
\hline
\end{tabular}




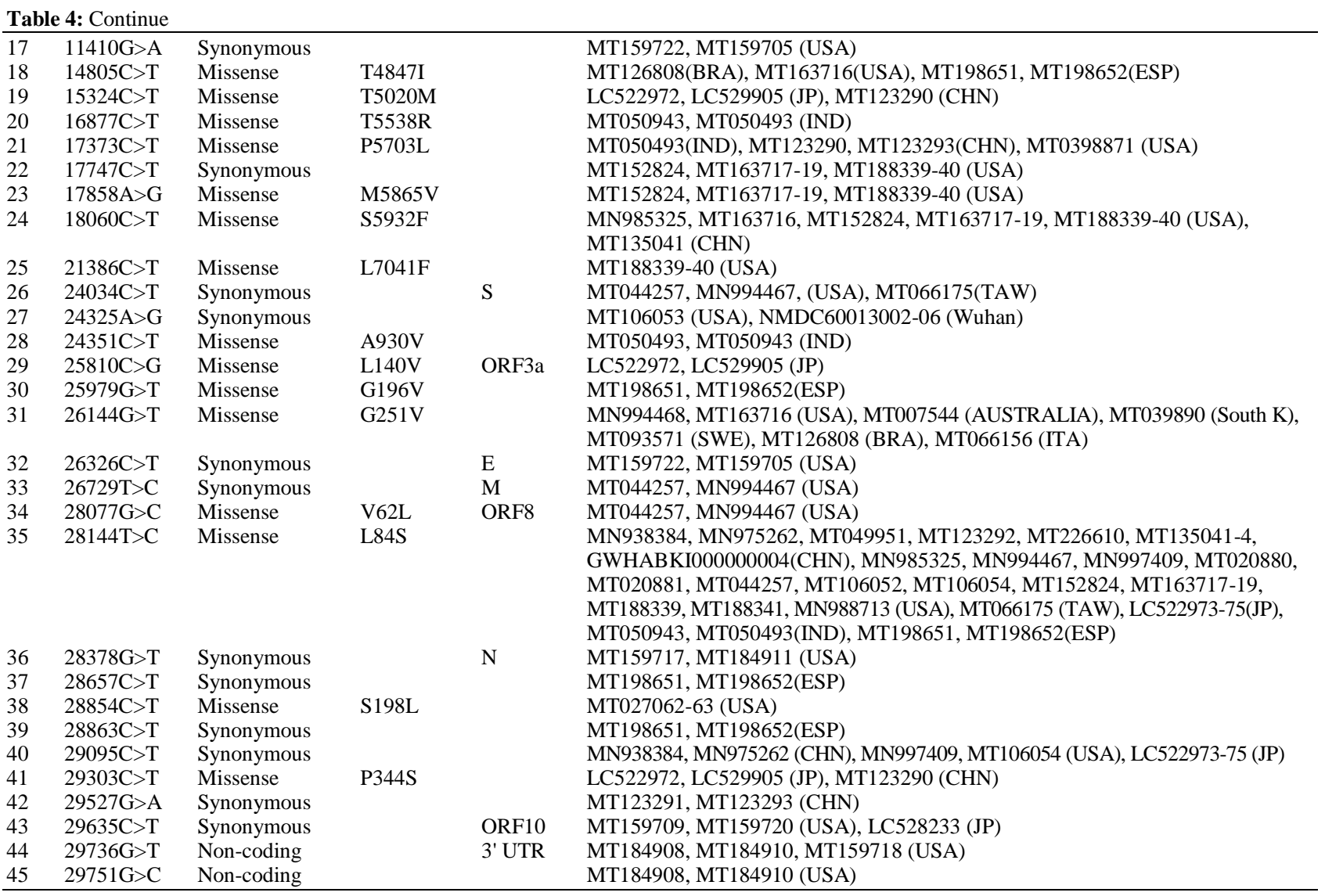

Table 5: Length, country and accession number of COVID-19 genome sequences used in this study

\begin{tabular}{|c|c|c|c|c|}
\hline$\#$ & Country & Length & Database & Accession number \\
\hline 1 & Wuhan & 29903 & Genbank & MN908947 \\
\hline 2 & China & 29838 & Genbank & MN938384 \\
\hline 3 & China & 29891 & Genbank & MN975262 \\
\hline 4 & USA & 29882 & Genbank & MN985325 \\
\hline 5 & USA & 29882 & Genbank & MN988713 \\
\hline 6 & USA & 29882 & Genbank & MN994467 \\
\hline 7 & USA & 29883 & Genbank & MN994468 \\
\hline 8 & USA & 29882 & Genbank & MN997409 \\
\hline 9 & Finland & 29806 & Genbank & MT020781 \\
\hline 10 & USA & 29882 & Genbank & MT020880 \\
\hline 11 & USA & 29882 & Genbank & MT020881 \\
\hline 12 & Japan & 29848 & Genbank & LC521925 \\
\hline 13 & USA & 29882 & Genbank & MT027062 \\
\hline 14 & USA & 29882 & Genbank & MT027063 \\
\hline 15 & USA & 29882 & Genbank & MT027064 \\
\hline 16 & Taiwan & 29870 & Genbank & MT066175 \\
\hline 17 & Japan & 29878 & Genbank & LC522973 \\
\hline 18 & Japan & 29878 & Genbank & LC522974 \\
\hline 19 & Japan & 29878 & Genbank & LC522975 \\
\hline 20 & Japan & 29878 & Genbank & LC522972 \\
\hline 21 & USA & 29879 & Genbank & MT039887 \\
\hline 22 & USA & 29858 & Genbank & MT044258 \\
\hline 23 & USA & 29882 & Genbank & MT044257 \\
\hline 24 & South Korea & 29903 & Genbank & MT039890 \\
\hline 25 & China & 29903 & Genbank & MT049951 \\
\hline 26 & Taiwan & 29870 & Genbank & MT066176 \\
\hline 27 & Nepal & 29811 & Genbank & MT072688 \\
\hline 28 & China & 29860 & Genbank & MT093631 \\
\hline
\end{tabular}




\section{Table 5: Continue}

\begin{tabular}{|c|c|c|c|c|}
\hline 29 & Sweden & 29886 & Genbank & МT093571 \\
\hline 30 & USA & 29882 & Genbank & MT106052 \\
\hline 31 & USA & 29882 & Genbank & MT106053 \\
\hline 32 & USA & 29882 & Genbank & MT106054 \\
\hline 33 & USA & 29882 & Genbank & MT118835 \\
\hline 34 & China & 29882 & Genbank & MT123291 \\
\hline 35 & China & 29891 & Genbank & MT123290 \\
\hline 36 & Japan & 29902 & Genbank & LC528233 \\
\hline 37 & Japan & 29902 & Genbank & LC528232 \\
\hline 38 & USA & 29878 & Genbank & MT152824 \\
\hline 39 & Brazil & 29876 & Genbank & MT126808 \\
\hline 40 & USA & 29903 & Genbank & MT163716 \\
\hline 41 & China & 29903 & Genbank & MT135041 \\
\hline 42 & China & 29903 & Genbank & MT135042 \\
\hline 43 & China & 29903 & Genbank & MT135043 \\
\hline 44 & China & 29903 & Genbank & MT135044 \\
\hline 45 & USA & 29897 & Genbank & MT163717 \\
\hline 46 & USA & 29903 & Genbank & MT163718 \\
\hline 47 & USA & 29903 & Genbank & MT163719 \\
\hline 48 & India & 29851 & Genbank & MT050493 \\
\hline 49 & India & 29854 & Genbank & MT012098 \\
\hline 50 & USA & 29882 & Genbank & MT159717 \\
\hline 51 & USA & 29882 & Genbank & MT159718 \\
\hline 52 & USA & 29882 & Genbank & MT159719 \\
\hline 53 & USA & 29882 & Genbank & MT159720 \\
\hline 54 & USA & 29882 & Genbank & MT159721 \\
\hline 55 & USA & 29882 & Genbank & MT159722 \\
\hline 56 & USA & 29882 & Genbank & MT159705 \\
\hline 57 & USA & 29882 & Genbank & MT159706 \\
\hline 58 & USA & 29882 & Genbank & MT159710 \\
\hline 59 & USA & 29882 & Genbank & MT159707 \\
\hline 60 & USA & 29882 & Genbank & MT159708 \\
\hline 61 & USA & 29882 & Genbank & MT159709 \\
\hline 62 & USA & 29882 & Genbank & MT159711 \\
\hline 63 & USA & 29882 & Genbank & MT159712 \\
\hline 64 & USA & 29882 & Genbank & MT159713 \\
\hline 65 & USA & 29882 & Genbank & MT159714 \\
\hline 66 & USA & 29882 & Genbank & MT159715 \\
\hline 67 & USA & 29882 & Genbank & MT159716 \\
\hline 68 & China & 29923 & Genbank & MT123292 \\
\hline 69 & China & 29871 & Genbank & MT123293 \\
\hline 70 & Italy & 29867 & Genbank & MT066156 \\
\hline 71 & Japan & 29903 & Genbank & LC529905 \\
\hline 72 & USA & 29882 & Genbank & MT184907 \\
\hline 73 & USA & 29880 & Genbank & MT184908 \\
\hline 74 & USA & 29882 & Genbank & MT184909 \\
\hline 75 & USA & 29882 & Genbank & MT184910 \\
\hline 76 & USA & 29882 & Genbank & MT184911 \\
\hline 77 & USA & 29882 & Genbank & MT184912 \\
\hline 78 & USA & 29882 & Genbank & MT184913 \\
\hline 79 & USA & 29783 & Genbank & MT188339 \\
\hline 80 & USA & 29845 & Genbank & MT188340 \\
\hline 81 & USA & 29835 & Genbank & MT188341 \\
\hline 82 & USA & 29829 & Genbank & MT192765 \\
\hline 83 & Taiwan & 29862 & Genbank & MT192759 \\
\hline 84 & Vietnam & 29891 & Genbank & MT192772 \\
\hline 85 & Vietnam & 29891 & Genbank & MT192773 \\
\hline 86 & Spain & 29611 & Genbank & MT198651 \\
\hline 87 & Spain & 29782 & Genbank & MT198652 \\
\hline 88 & China & 29899 & Genbank & MT226610 \\
\hline 89 & Pakistan & 29836 & Genome Warehouse & GWHACDD01000001 \\
\hline 90 & Wuhan & 29899 & Genome Warehouse & GWHABKF0000000001 \\
\hline
\end{tabular}




\begin{tabular}{|c|c|c|c|c|}
\hline 91 & Wuhan & 29889 & Genome Warehouse & GWHABKF0000000003 \\
\hline 92 & Wuhan & 29890 & Genome Warehouse & GWHABKF0000000004 \\
\hline 93 & Wuhan & 29891 & NMDC & NMDC60013002-06 \\
\hline 95 & Wuhan & 29891 & NMDC & NMDC60013002-08 \\
\hline 96 & Wuhan & 29896 & NMDC & NMDC60013002-09 \\
\hline 97 & Wuhan & 29891 & NMDC & NMDC60013002-10 \\
\hline 99 & Wuhan & 29903 & Genbank & NC_045512 \\
\hline 100 & China & 29871 & Genbank & MN996530 \\
\hline 101 & China & 29894 & Genbank & MN996528 \\
\hline 102 & India & 29874 & Genbank & МT050943 \\
\hline
\end{tabular}

$\begin{array}{ll}354 & 403\end{array}$

MT044258-USA CGTGGCTTTG GAGACTCCGT GGAGGAGGTC TTATCAGAGG CACGTCAACA

MT188341-USA CGTGGCTTTG GAGACTCCGT GGAGGAGGTC TTATCAGAGG CACGTCAACA

MT159716-USA CGTGGCTTTG GAGACTCCGT GGAGGAGGTC TTATCAGAGG CACGTCAACA LC521925-JP CGTGG

500

MT044258-USA GCACCTCA MT188341-USA GCACCTCATG GTCATGTTAT GGTTGAGCTG GTAGCAGAAC TCGAAGGCAT MT159716-USA GCACCTCA LC521925-JP GCACCTCATG GTCATGTTAT GGTTGAGCTG GTAGCAGAAC TCGAAGGCAT MT188341-USA AAAGGAGCTG GTGGCCATAG TTACGGCGCC GATCTAAAGT CATTTGACTT MT159716-USA AAAGGAGCTG GTGGCCATAG TTACGGCGCC GATCTAAAGT CATTTGACTT LC521925-JP AAAGGAGCTG GTGGCCATAG TTACGGCGCC GATCTAAAGT CATTTGACTT

(A) 3 In-frame deletions at positions: $\mathrm{C}_{359}-\mathrm{T}_{382}, \mathrm{~T}_{508}-\mathrm{T}_{522} \& \mathrm{~A}_{686}-\mathrm{T}_{694}$

20261 20310

MT050943-IND TAGAATTAGC TATGGATGAA TTCATTGAAC GGTATAAATT AgAagGCTAT MT012098-IND TAGAATTAGC TATGGATGAA TTCATTGAAC GGTATAAATT AGAAGGCTAT MT044258-USA TAGAATTAGC TATGGATGAA TTCATTGAAC GGTATAAATT AGAAGGCTAT MT039887-USA TAGAATTAGC TATGGATGAA TTCATTGAAC GGTATAAA־GAAGGCTAT 21951

MT050943-IND AAGTCTGTGA ATTTCAATTT TGTAATGATC CATTTTTGGG TGTTTATTAC MT012098-IND AAGTCTGTGA ATTTCAATTT TGTAATGATC CATTTTTGGG TGTTTA---C MT044258-USA AAGTCTGTGA ATTTCAATTT TGTAATGATC CATTTTTGGG TGTTTATTAC MT039887-USA AAGTCTGTGA ATTTCAATTT TGTAATGATC CATTTTTGGG TGTTTATTAC

(B) 2 Out-of-frame deletions in MT039887-USA \& MT012098-IND 21350

MT044258-USA GGAGGAATAC AAATCCAATT CAGTTGTCTT CCTATT---C TTTATTTGAC MT188341-USA GGAGGAATAC AAATCCAATT CAGTTGTCTT CCTATTTCTГ TTTATTTGAC MT159716-USA GGAGGAATAC AAATCCAATT CAGTTGTCTT CCTATT---C TTTATTTGAC LC521925-JP GGAGGAATAC AAATCCAATT CAGTTGTCTT CCTATT---C TTTATTTGAC (C) 1 Insertion in MT188341-USA

Fig. 1: In -frame, out-of-frame deletions and an insertion in USA, Japanese and Indian variants 


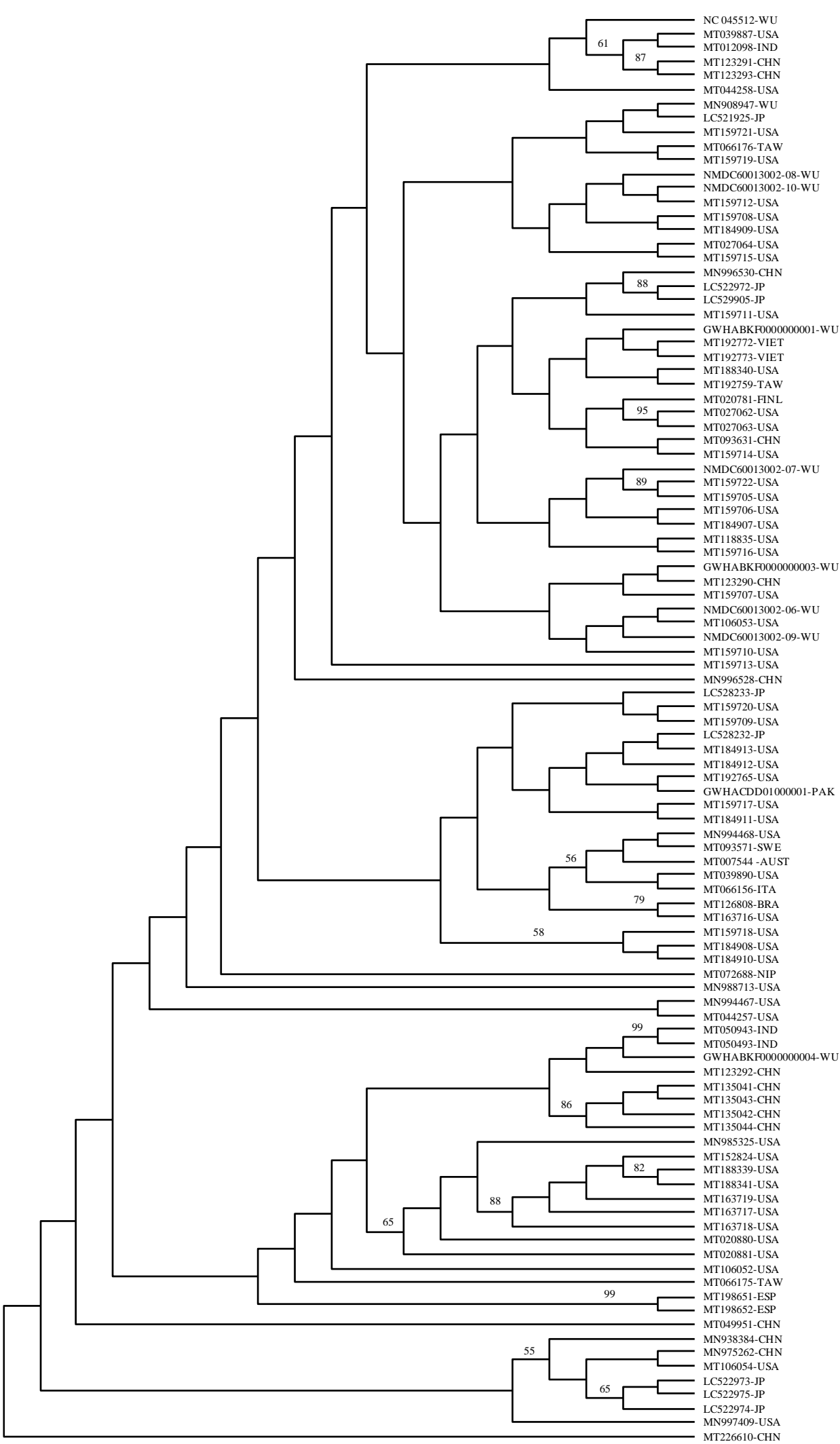

Fig. 2: NJ tree constructed by using 29532 sites of the collected genomes. Bootstrap values are shown at nodes whenever they are above $50 \%$ 


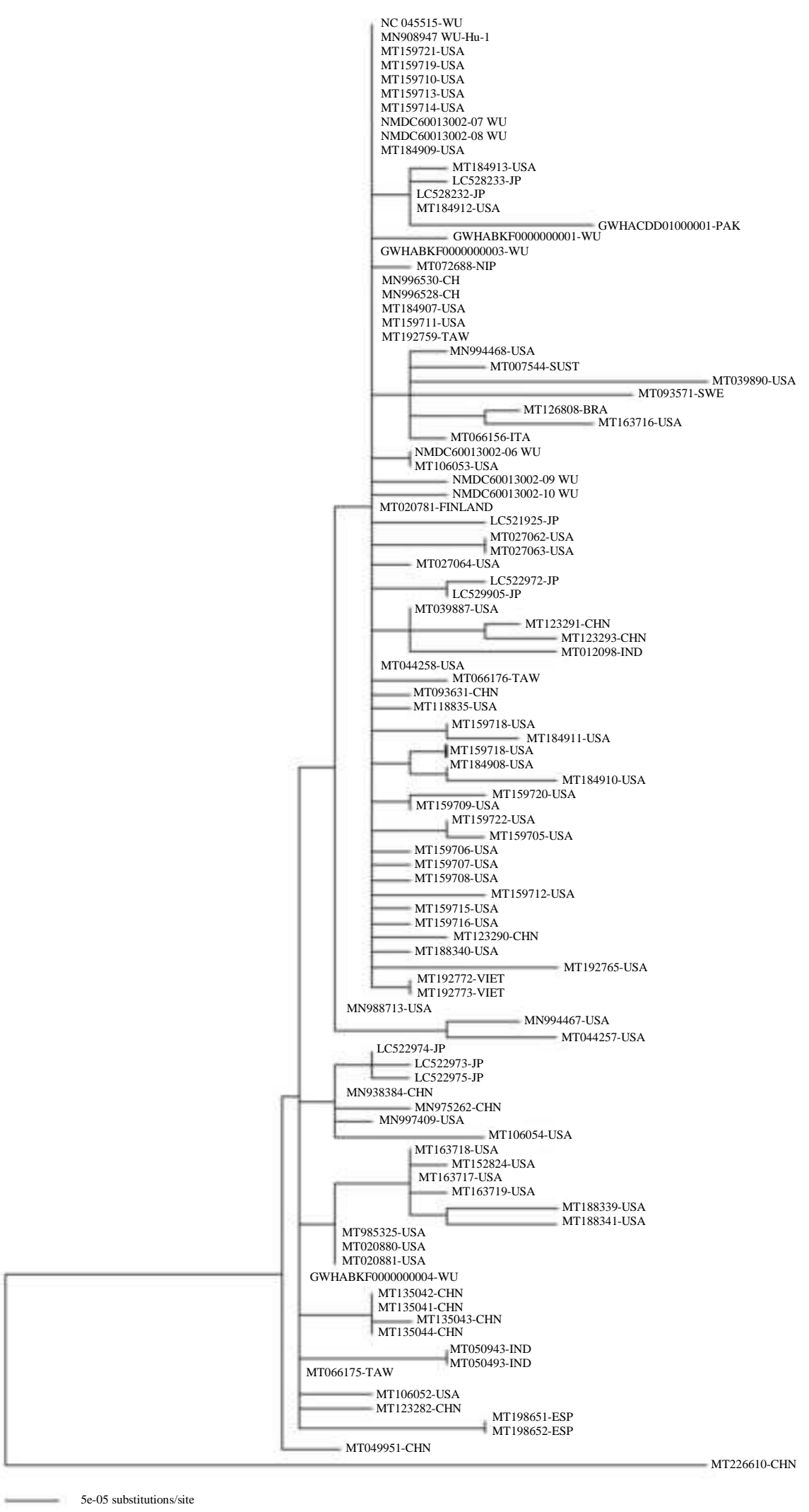

Fig. 3: ML phylogenetic tree constructed by the dataset using the modeltest GTR + I + G 
For the 101 missense variants, 80 variants are found in the longest ORF1ab gene distributed in the cleaved nonstructural proteins (NSP1-NSP16). However, more variants are found in the structural protein genes $(\mathrm{S}$, ORF3a and N). MT226610-CHN was the fastest evolving substrain as it exhibited 29 substitutions. One of the out-of-frame-deletions is found close to 3 'end of nsp 15 protein of an Indian substrain and the other one is found close to 5'end of S protein of the USA strain (Fig. 1). The first mutation probably did not alter the O-ribose methyltransferase (nsp16) since it is located at the end of the gene, while the second could alter the posttranslational spike, glycoprotein. This mutation may increase disease susceptibility (Zimmerman et al., 1997) or stop protein function indicating that it is not necessary for efficient viral transmission. It is not known that $\mathrm{S}$ deletion enhances virulence or transmission rates of the virus and it is not known whether the strain acquiring this deletion could successfully transmit to a new host (Assiri et al., 2016).

Fortunately, this study collected various COVID-19 genomes from the same place of origin as shown in Table 5 (51 genomes from USA, 25 from China (including 10 from Wuhan) and 8 from Japan). This supports that the novel mutations found herein could reflect the diversity of the place of origin rather being acquired during spreading of the infection (Matsuda et al., 2020). It is therefore an indication that stopping virus outbreak is possible in the short-term future. However, the constructed tree (Fig. 2) indicated that viruses from the same country did not form a single group, which suggests that CoVs-19 were introduced to each country several times (Koyama et al., 2020; Matsuda et al., 2020) and it, thus, may be difficult to follow the virus origin. The phylogeny of the maximum-likelihood analysis (Fig. 3) indicated a possible transmission scenario of the virus. The tree referred to Chinese origin of COVID-19 and showed its transmissions to USA, Spain, Japan and India.

Researchers sequenced a lot of SARS-CoV-2 genomes and shared results during the pandemic. The sequenced data allowed public health officials to evaluate the relevant epidemiological parameters such as the reproductive number and virus introduction into new regions. Knowing the possibilities for the outbreak is still managing health precautionary measures which could be conducted in daily life (Hopkins, 2020). Understanding genetic framework of COVID-19 genome enhances WHO's ability to analyze the risk of the virus introduction into countries and define the response actions and prioritization of resources, as well as the possible capacity to manage the virus outbreak. The implementation of action plans for health security is occurring globally with varied progress rates (Samhouri et al., 2018) and is actively supported by WHO to enhance operational readiness for the virus in countries (Al-Mandhari et al., 2020).

\section{Conclusion}

In conclusion, the virus is still considered a threat to human health security as there is lack of knowledge about the origin and the reasons for its outbreak. Chinese origin could be possible. Two debates about the virus outbreak are either the diversity of the place of origin or spreading the infection through individuals' movements between countries. Emergence of new variants by releasing more genomes could help in clarifying the virus origin, the reasons of its outbreak and the development of vaccines or effective precautions.

\section{Acknowledgment}

The author would like to express his deep thanks to the Vice Presidency for Scientific Research at Naif Arab University for Security Sciences for their kind encouragement of this work.

\section{Funding Information}

This work was supported by Security Research Center at Naif Arab University for Security Sciences.

\section{Ethics}

This article is original and contains unpublished material.

\section{Conflict of Interest}

The author declares that he has no conflict of interest. No ethical approval for this study is needed since it depended on the data deposited in the Genbank database.

\section{References}

Al-Mandhari, A., Samhouri, D., Abubakar, A., \& Brennan, R. (2020). Coronavirus Disease 2019 outbreak: preparedness and readiness of countries in the Eastern Mediterranean Region. https://coronavirus. 1science. com/item/6166e6044a2114454e136505bc74865c1c $52 \mathrm{e} 8 \mathrm{da}$.

Assiri, A.M., Biggs, H.M., Abedi, G.R., Lu, X., Bin Saeed, A., Abdalla, O., Mohammed, M., Al-Abdely, H.M., Algarni, H.S., Alhakeem, R.F., \& Almasri, M.M. (2016), May. Increase in Middle East respiratory syndrome-coronavirus cases in Saudi Arabia linked to hospital outbreak with continued circulation of recombinant virus, July 1-August 31, 2015. In Open forum Infect Diseases (Vol. 3, No. 3), Oxford University Press.

Cascella, M., Rajnik, M., Cuomo, A., Dulebohn, S. C., \& Di Napoli, R. (2020). Features, evaluation and treatment coronavirus (COVID-19). In Statpearls [internet], StatPearls Publishing. 
Chan, J. F. W., Kok, K. H., Zhu, Z., Chu, H., To, K. K. W., Yuan, S., \& Yuen, K. Y. (2020). Genomic characterization of the 2019 novel humanpathogenic coronavirus isolated from a patient with atypical pneumonia after visiting Wuhan. Emerg Microb \& Infect, 9(1), 221-236.

Chan, J. F. W., To, K. K. W., Tse, H., Jin, D. Y., \& Yuen, K. Y. (2013). Interspecies transmission and emergence of novel viruses: lessons from bats and birds. Trends In Microbiol, 21(10), 544-555.

Chen, Y., \& Guo, D. (2016). Molecular mechanisms of coronavirus RNA capping and methylation. Virol Sin, 31(1), 3-11.

Chen, Y., Liu, Q., \& Guo, D. (2020). Emerging coronaviruses: genome structure, replication and pathogenesis. J Med Virol, 92(4), 418-423.

Ge, X.Y., Li, J.L., Yang, X.L., Chmura, A.A., Zhu, G., Epstein, J.H., Mazet, J.K., Hu, B., Zhang, W., Peng, C., \& Zhang, Y.J. (2013). Isolation and characterization of a bat SARS-like coronavirus that uses the ACE2 receptor. Nature, 503(7477), 535-538.

Hall, T., Biosciences, I., \& Carlsbad, C. (2011). BioEdit: an important software for molecular biology. GERF Bull Biosci, 2(1), 60-61.

Hopkins, J. (2020). SARS-CoV-2 Genetics. Center for Health Security. https://www.centerforhealthsecurity.org/resources/C OVID-19/COVID-19-fact-sheets/200128-nCoVwhitepaper.pdf

Koyama, T., Platt, D., \& Parida, L. (2020). Variant analysis of SARS-CoV-2 genomes. Bull World Health Organization, 98(7), 495.

Maddison, W. P., \& Maddison, W. P. (2002). Macclade. sunderland. MA: sinauer Associates.

Matsuda, T., Suzuki, H., \& Ogata, N. (2020). Phylogenetic analyses of the severe acute respiratory syndrome coronavirus 2 reflected the several routes of invasion in Taiwan, the United States and Japan. arXiv preprint arXiv:2002.08802.
Nguyen, T. M., Zhang, Y., \& Pandolfi, P. P. (2020). Virus against virus: a potential treatment for 2019nCov (SARS-CoV-2) and other RNA viruses.

Paul, S. M. (2006). The molecular biology of coronavirus. Adv Virus Res, 66(48), 193-292.

Perlman, S., \& Netland, J. (2009). Coronaviruses postSARS: update on replication and pathogenesis. Nature Rev Microbiol, 7(6), 439-450.

Saitou, N., \& Nei M (1987). The neighbor-joining method: A new method for reconstructing phylogenetic trees. Mol Biol Evol, 4, 406-425.

Samhouri, D., Ijaz, K., Rashidian, A., Chungong, S., Flahault, A., Babich, S. M., \& Mahjour, J. (2018). Analysis of Joint External Evaluations in the WHO Eastern Mediterranean Region. East Mediterr Health J, 24(5), 477-87.

Swofford, D. L. (2002). PAUP: phylogenetic analysis using parsimony, version $4.0 \mathrm{~b} 10$.

Tamura, K., \& Nei, M. (1993). Estimation of the number of nucleotide substitutions in the control region of mitochondrial DNA in humans and chimpanzees. Mol Biol Evol, 10(3), 512-526.

Wang, L. F., Shi, Z., Zhang, S., Field, H., Daszak, P., \& Eaton, B. T. (2006). Review of bats and SARS. Emerg Infect Diseases, 12(12), 1834.

WHO. (2020). Coronavirus disease 2019 (COVID-19): situation report, 82 .

Zimmerman, P.A., Buckler-White, A., Alkhatib, G., Spalding, T., Kubofcik, J., Combadiere, C., Weissman, D., Cohen, O., Rubbert, A., Lam, G., \& Vaccarezza, M. (1997). Inherited resistance to HIV-1 conferred by an inactivating mutation in $\mathrm{CC}$ chemokine receptor 5: studies in populations with contrasting clinical phenotypes, defined racial background and quantified risk. Mol Med, 3(1), 23-36. 